\title{
The luteo-placental shift, the guardian of pre-natal life
}

\author{
ARPAD CSAPo \\ M.D. \\ The Department of Obstetrics and Gynecology, Washington University School of Medicine, \\ St Louis, Missouri
}

\section{Introduction}

The 'luteo-placental shift' is defined here as a shift in progesterone synthesis during pregnancy from the corpus luteum to the placenta. Distinct changes in the regulation of uterine function follow. It is predicted that this shift in the anatomical site of hormone support, from an extra- to an intra-uterine location, changes the distribution of progesterone, the character of the myometrial block and, therefore, uterine activity and reactivity. In different species the shift seems to occur at different times in gestation and to different degrees. It is these variations in the timing and the degree of the shift which best explain the noted 'species differences' in uterine activity. If so, 'species differences' are superimposed modifications, rather than fundamental alterations, of the basic regulatory mechanism of uterine function.

The purpose of the shift is thought to be the protection of pregnancy beyond the life span of the corpus luteum, thus allowing continued pre-natal development. However, by weakening the progesterone block, the shift often permits a gradual early 'evolution' of uterine activity and reactivity long before term. If this 'evolution process' oversteps physiological limits a 'hostile' contractile environment develops, exposing the foetus to considerable hazards and, in extreme conditions, to prematurity and pre-natal defects. The experiments to be reported examine the merits of these concepts in the human, the rat and the rabbit.

A critical survey of the current literature (Csapo \& Wood, 1968) revealed that several maternal and foetal factors are implicated in the complex regulatory mechanism in control of uterine function during gestation and at labour. However, the contributions of these factors appear to differ in significance. The 'luteo-placental unit' dominates uterine function, for when its influence is complete it cannot be overruled by the others. Experiences with foetal death in utero and with the surgical separation of the 'foeto-placental unit', reveal that it is the placental, rather than the foetal component, which affects the myometrium directly. Foetal death has no acute, but only a chronic, influence on the continuation of pregnancy. The duration of gestation with a dead foetus seems to depend on the relationship between residual placental function and uterine volume. Within the 'luteo-placental unit' the placental component appears to be dominant, for it is indispensable in all species, while the luteal component is not. Even the rabbit, the champion of luteal dominance, requires placental support for the sustenance of the corpus luteum, an effect readily substituted for by estrogen therapy (Porter, Becker \& Csapo, 1968).

It is proposed to describe results obtained by multi-disciplinary teamwork in a series of experiments undertaken in the human female, the rat and rabbit in collaboration with a number of investigators in my laboratory: Drs Abe, Kerenyi, Ogata, PintoDantas, Porter and Takeda, and in the steroidchemical laboratory of Dr Wiest.

\section{Clinical trials}

Extensive and carefully controlled observations were carried out in twenty-six non-pregnant patients with normal menstrual cycles. Intra-uterine pressure changes were recorded by means of small balloons attached to catheters and inserted into the uterine cavity through the vagina (Csapo et al., 1966). Spontaneous activity and the response to intravenous oxytocin were recorded sequentially through the menstrual cycle. During the menstrual flow regular pressure cycles of high amplitude and low frequency were recorded. After the cessation of the menstrual flow the pressure decreased while the frequency increased. On day 16 the pressure cycles were of minimum amplitude and high frequencyfeatures characteristic of a 'blocked' uterus. This condition of suppressed activity prevailed until about day 26 when the pressure cycles increased somewhat. A marked increase in pressure occurred during the last day of the cycle. The quantitative difference between the recorded pressure at day 2 and at other stages of the cycle was statistically significant* at the

* For biostatistical analysis the authors are grateful to Dr. J. A. Hagans, Dr. C. A. Schlagel and Dr. J. A. Soda of The Upjohn Company, Kalamazoo, Michigan. 


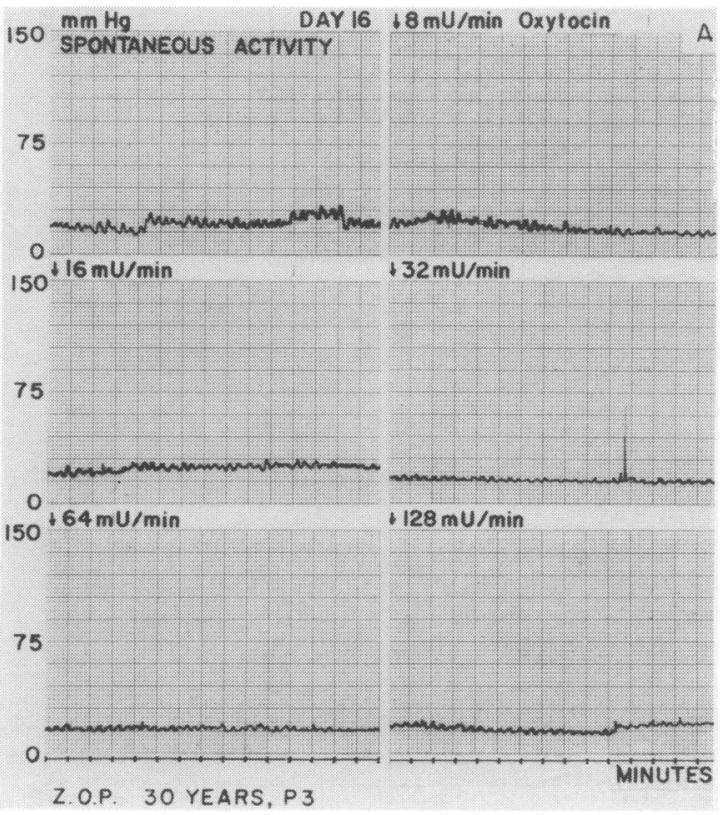

FIG. 1 (A). The spontaneous activity and oxytocin response of the human uterus at day 16 of the normal menstrual cycle. Intra-uterine pressure is measured by the 'demobilized microballoon' method.

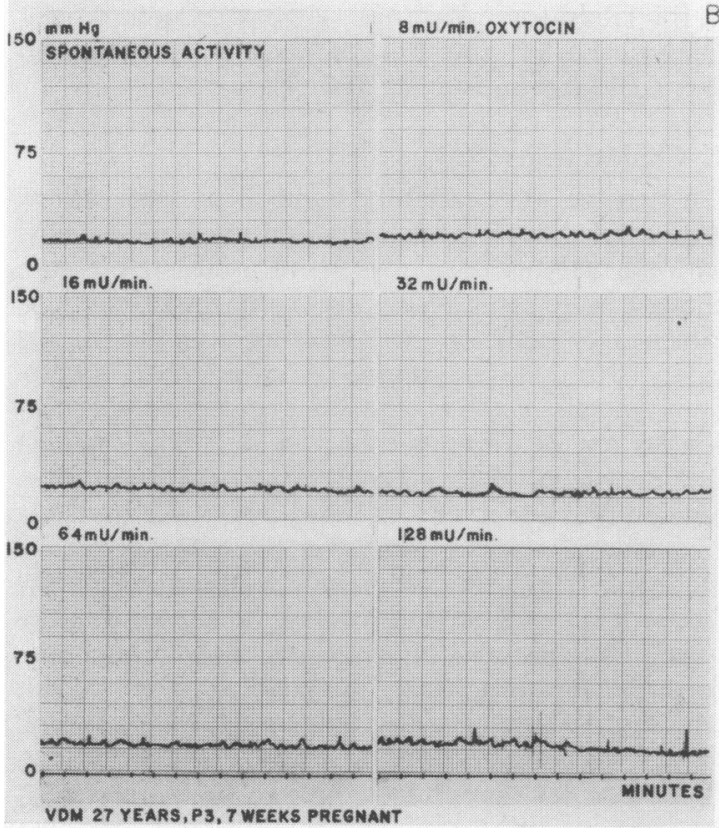

FIG. 1 (B). The spontaneous activity and oxytocin response of the human uterus during the 7 th week of normal pregnancy. Intra-uterine pressure is measured by the 'demobilized microballoon' method. Note that as during the normal mid-cycle there is no response to oxytocin in doses up to $128 \mathrm{mU} / \mathrm{min}$.

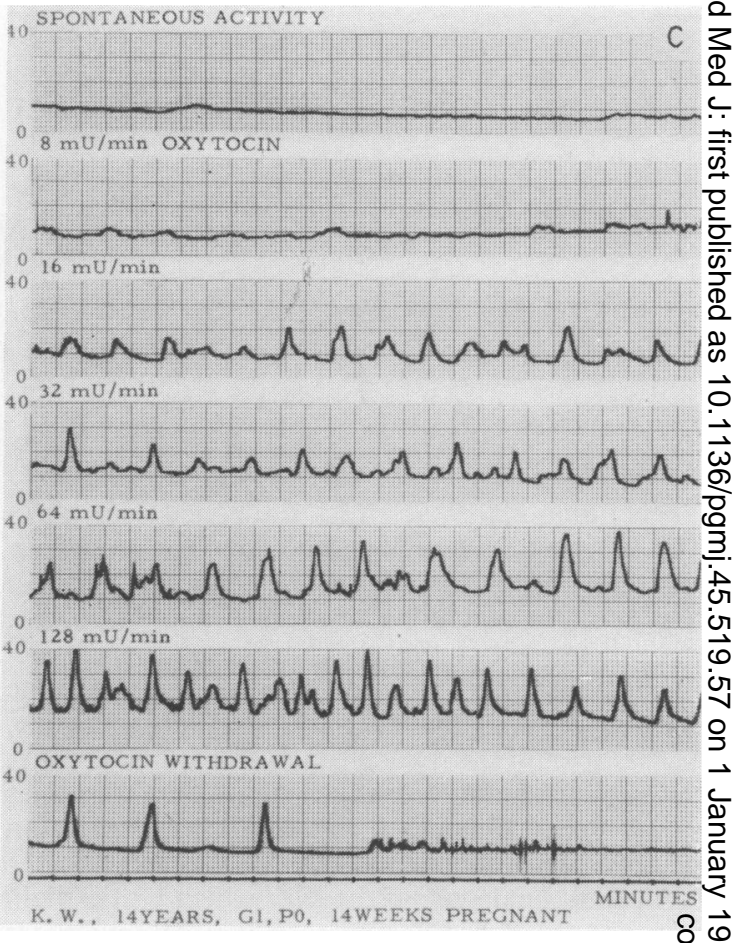

FIG. 1 (C). The spontaneous activity and oxytocint response of the human uterus during the 14th week of normal pregnancy. Intra-uterine pressure is measure by the 'transabdominal method'. Note the distinct, but limited, response to oxytocin.

level of $P<0.01$, but not between days 16-17 and $\stackrel{\mathbb{2}}{\rightarrow}$ 26. The changes were paralleled by the oxytocino response, which was distinct during the menstrual ${ }_{\vec{J}}$ flow and subsequently gradually disappeared Characteristically, there was no oxytocin response during the secretory phase, irrespective of the dose 3 and the method of administration (Fig. 1). Occasion-:ally a slight increase in resting pressure was recorded 3 . By day 26 an abortive response could again be obtained which increased at the end of the cycle.

These observations are in harmony with theo findings (Van der Molen \& Groen, 1965; Runne-? baum \& Zander, 1967; Johansson, Neill \& Knobil,o 1968) that a rise in plasma progesterone precedes ovulation by several days and declines sharply ato the end of the cycle. Furthermore, progesteroner treatment reduces the peak pressure of spontaneous $\mathrm{C}_{\mathrm{W}}$ activity found at the beginning of the cycle andodelays its evolution at the end (Csapo et al., 1966) Thus in the non-pregnant human female the behaviour of the myometrium near and after the mid-o cycle is in accordance with the ovarian support of the progesterone block, characterized in the pregnantō rabbit. 
During the 7th week of pregnancy five obstetrically normal patients were studied in collaboration with Pinto-Dantas by the same method used in non-pregnant patients. No oxytocin response could be obtained, even with infusion rates of $128 \mathrm{mU} / \mathrm{min}$ (Fig. 1). However, when intra-uterine pressure was recorded transabdominally in patients more than 14 weeks pregnant (Bengtsson \& Csapo, 1962), it was found that a response to oxytocin could always be obtained, though massive doses $(128 \mathrm{mU} / \mathrm{min})$ were required in the second trimester to induce $18 \mathrm{mmHg}$ pressure (Fig. 1). In the 36th week, the spontaneous pressure cycles reached $18 \mathrm{mmHg}$ and $8 \mathrm{mU} / \mathrm{min}$ oxytocin induced an average $37 \mathrm{mmHg}$ pressure. At 40 weeks spontaneous and oxytocininduced pressure cycles were 32 and $65 \mathrm{mmHg}$, respectively (Csapo \& Sauvage, 1968).

Wilson (1937) demonstrated by clinical observations in eleven patients that at the 7 th week of gestation the ovaries are 'indispensable' to the maintenance of pregnancy as a rule. This indicates that a certain degree of placental 'maturity' is a prerequisite of the luteo-placental shift and that at the 7th week this maturity is not reached invariably. Thus the described observations of oxytocin response fall into place. They reveal that so long as the ovaries are the source of progesterone, the response of the myometrium to oxytocin may be completely suppressed. After the luteo-placental shift, however, this no longer holds and there is always a response, though very high doses of oxytocin may be required for a partial response.

\section{Experiments in pregnant rats}

A report, describing physiological, electro-physiological and some of the steroid chemical observations has been presented (Csapo, 1967). A brief summary of these results will be sufficient here in providing background for the description of electrophysiological experiments.

Holtzman-bred Sprague-Dawley rats, 80-90 days old and 200-230 $\mathrm{g}$ weight were used. The time of mating was determined with $\pm 3 \mathrm{hr}$ accuracy. The series consisted of 647 rats and the data relating to their pregnancies were carefully documented. The average litter size was ten, $94 \%$ of the litters consisting of six to fourteen foetuses. In over $80 \%$ of the mothers the foetuses were unequally distributed between the two horns. Between the 13th and 22nd days of pregnancy, the foetal and placental weight at a given time in gestation was closely similar in the different animals with an average standard deviation of less than $3 \%$. Thirty-two control animals were allowed to go to term and, of these, thirty delivered on the morning of the 22nd day while two, which had more than twelve foetuses, delivered the previous night. Delivery was completed in about $30 \mathrm{~min}$.

Ovariectomy before day 14 (5-6 days after implantation) resulted in rapid death and resorption of the litter. This was not surprising (in view of Wilson's clinical study, 1937) in that at day 13 the rat placenta is $0.11 \mathrm{~g}$, only $20 \%$ of the 'mature' value $(0.55 \pm 0.01 \mathrm{~g}$ at day 20$)$. After the 13th day the placental weight increases rapidly, as does the success of the ovariectomized animal in maintaining pregnancy. Thus ovariectomy at 16-17 days resulted in over $60 \%$ foetal survival at day 21 . This observation provides evidence that, in the rat, the ovary is dispensable towards the end of pregnancy to a considerable degree, at least under the conditions of our experiments, conducted between September and April.

The success of the ovariectomized animal in maintaining pregnancy was distinctly dependent on placental hypertrophy. If placental hypertrophy did not occur, premature delivery started on the 19th to 20th day. A group of twenty-four ovariectomized animals with intact pregnancy had an average placental weight of $0.65 \pm 0.02 \mathrm{~g}$, while thirty-three animals in premature delivery $0.56 \pm 0.01 \mathrm{~g}$. This difference, documented by examining 495 placentae, is highly significant $(P<0.001)$.

The character of this premature delivery appeared to be of considerable theoretic interest, in that:

(1) It started from the uterine horn of greater volume, as a rule.

(2) Only a limited number of foetuses were discharged at long intervals and their placentae were retained.

(3) Delivery was interrupted and prolonged for hours or days, allowing a fraction of the litter to reach term.

Thus by discharging a number of foetuses and retaining the placentae, the animal apparently 'turned off' myometrial activity and still remained pregnant. As the uterine volume increased with the continued growth of the remaining foetuses, however, it became optimal again for 'turning on' activity and another foetus was delivered. In this way a protracted delivery was achieved. However, neither placental hypertrophy, nor placental retention (and the lowering of the foetal/placental ratio) provided complete protection of the foetuses which went to term, for the incidence of foetal defects was marked. Deformities, haemorrhage, oedema, missing extremities and hydramnios were frequent, especially in the two foetuses located at the cervical end of the horn. Those foetuses which did reach term without marked defects were unusually mature.

The development of a 'hostile' uterine environment after ovariectomy was readily prevented by 'sustained' progesterone replacement therapy. The 
effective dose was high, relative to that required by ovariectomized rabbits, for a daily dose of less than $2 \mathrm{mg}$ did not always offer $100 \%$ protection. However, at $2 \mathrm{mg} /$ day and higher dose levels, pregnancy was maintained even if ovariectomy was performed before the 15th day. It was found that if ovariectomy was carried out on days 14-15 of pregnancy, 'decremental' progesterone therapy (sequential daily doses of $4,3,2,1.5,1.0$ and $0.5 \mathrm{mg}$ ) was also effective in maintaining pregnancy to term, despite the fact that the treatment was discontinued 3 days before term. Both forms of progesterone replacement therapy promoted marked placental hypertrophy and increased the weight of the newborn. An independent study showed that gestagens, other than progesterone, are also effective (Van der Vies \& Feenstra, 1967). It was found unnecessary to add oestrogen to the progesterone therapy in order to achieve viable young, for the foetuses, delivered at term by hysterectomy, developed normally in the care of foster mothers. However, if $2 \mathrm{mg}$ or more of oestradiol were added to the daily doses of progesterone, then the placental hypertrophy was suppressed, while $10 \mathrm{mg} /$ day oestradiol suppressed even normal placental growth, resulting in premature delivery. In contrast, continued progesterone therapy, without oestrogen, prolonged gestation and delivery could not be induced with oxytocin at term.

Additional experiments (in fifty-one rats) were carried out on the 17th day of pregnancy. Both ovaries were removed and in one horn all the placentae were dislocated by gentle pressure between finger and thumb to move the whole conceptus sac sideways. After recovery, the animals were housed on a grating which allowed delivered foetuses and placentae to pass through onto the tray below. Twenty-three rats received $4 \mathrm{mg} /$ day progesterone, the remainder were left untreated. All were kept under close observation and killed 12-48 $\mathrm{hr}$ after the operation. The distribution of conceptuses in the uterine horns was carefully recorded both at the time of operation and at the time of autopsy. In the untreated rats, $75 \%$ of the dislocated conceptuses were aborted but only $4 \%$ of the intact foetuses. On the other hand, in those animals which were receiving progesterone treatment, only $8 \%$ of the dislocated conceptuses were aborted. Thus these experiments show that the complete removal of the 'systemic' luteal progesterone component, together with the unilateral removal of the 'local' placental component, creates a functional 'asymmetry' between the two uterine horns and the majority of foetuses are delivered from one horn but retained in the other. However, when the status quo of the 'systemic' progesterone is restored by daily injections of progesterone, the majority of foetuses are retained in both horns.
The precise electrophysiological conditions associ ated with this functional asymmetry of the myometrium was investigated in collaboration withe Dr H. Takeda. The study was carried out in eight: rats $48 \mathrm{hr}$ after ovariectomy and unilateral disloca tion of the placentae. Under ether anaesthesia theo abdomen was opened by midline incision and theo cervical ends of the two uterine horns were exposed $\overline{\bar{c}}$. Through a longitudinal slit in the vagina, a micro $\mathbb{\Omega}$ balloon $(0.8 \mathrm{ml}$ capacity), was inserted into eachw uterine horn and connected to a Statham pressures transducer by a polyethylene tube. The balloons were filled with water, and the vaginal wound closed $\vec{\overrightarrow{ }}$ Two 'suction' electrodes (Hoffman et al., 1959) were subsequently used for the recording of the electric activity of the two uterine horns. These electrodes consisted of a polyethylene tube (0.5 mme bore) surrounding an $\mathrm{Ag}-\mathrm{AgCl}$ wire $(0.1 \mathrm{~mm}$ diameter). These electrodes were placed over theo serosal surface of the various uterine regions and applied by vacuum suction so that the electrode tipo was in close contact with the myometrium during suction and was kept in place by it. When suction was discontinued the electrode was readily detached $\stackrel{0}{5}$ A spiral shaped 'reference' electrode was placed in the vagina. The electrodes were connected to an A.C. amplifier with a 2-sec time constant and the signalso were recorded simultaneously with the intra-uter pressure (by a Grass Polygraph). The exposiduterine regions were kept moist by covering the with sterile gauze soaked in warm saline and recordings were made in different regions of both horns.

The action potentials recorded by suction elec $\stackrel{\square}{\Rightarrow}$ trodes result from the potential difference created, between the transiently ischaemic (depolarized) 'suction region' and the nearby uterine portions Since these electrodes record the activity of groups of cells, rather than of one cell, the amplitude of the action potentials depends on the number of cells. which fire synchronously. The shape of the potentials. also depend on the electrical synchrony betweer cells (Hoffman et al., 1959).

The electrophysiological findings in the various areas of the uterus are illustrated by the origina? tracings of Fig. 2. In the experimental (placentas dislocated) horn, from which most of the foetuses had already been delivered, marked electricap activity was recorded in all areas and the train dis $N^{\circ}$ charges of the different myometrial regions were्य synchronous. Furthermore, electrical activity was regular and occurred synchronously with the intrauterine pressure of that horn. Such activity indicate that the myometrium has already escaped from the progesterone block (Csapo \& Takeda, 1965). Ino contrast to this horn in which the placentae ha\$ been dislocated $48 \mathrm{hr}$ previously, the control horro 


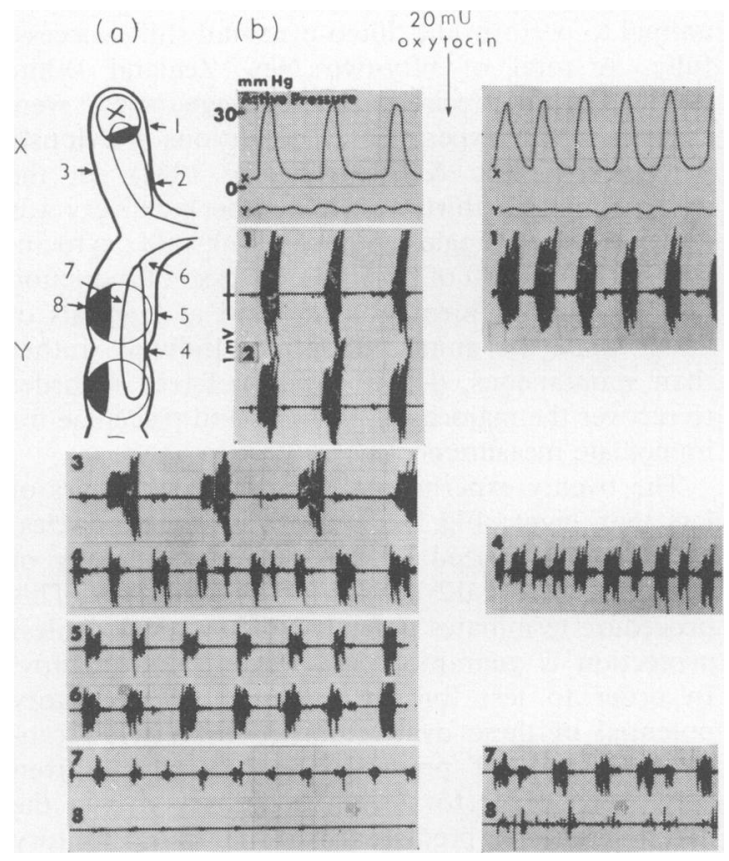

FIG. 2. The influence of the placenta on spontaneous and oxytocin-induced electrical and mechanical activity of the myometrium. On the 16th day of pregnancy in the rat, both ovaries were removed and, in one horn, the placentae were dislocated. The latter horn aborted its contents on day 17 and recordings were made on day 18 . (a) Diagram of the recording positions of the suction electrodes on the two horns of the uterus. X, Experimental horn which had aborted its contents; Y, intact horn. (b) Tracings of spontaneous pressure changes in the two horns and of electrical activity recorded from sites 1-8. The effect is shown of an intravenous injection of $20 \mathrm{mU}$ oxytocin on pressure and on sites $1,4,7$ and 8 . Note the synchronous electrical activity in the active aborted horn (1,2 and 3) and the high, regular waves of intra-uterine pressure associated with it (X). The interplacental region of the intact horn (4) displays distinct but irregular electrical activity and as the electrodes are moved closer to the cervix away from the lowermost placenta, electrical activity improves ( 5 and $6)$. In contrast, as the electrodes approach the placental bed, the train discharges gradually diminish (7 and 8 ). This asynchronous electrical activity is characteristic of the progesterone block and only permits irregular pressure cycles of limited magnitude. The effect of oxytocin is to improve both mechanical and electrical activity in the empty horn ( $X$ and 1 ). However, in the intact horn, although electrical activity is improved in the interplacental region (4), no distinct train discharges are triggered in the placental region (8) and, as a result, there is little improvement in the pressure cycles recorded in this horn $(\mathrm{Y})$. showed much less activity. There were, however, differences in electrical activity according to the distance of the recording site from the placenta. Thus when the electrodes were applied to the myometrium in an interplacental region, distinct but irregular electrical activity was recorded. As the electrodes were moved closer to the cervix and away from the lowermost placenta, electrical activity improved. When, on the other hand, electrodes approached the placental bed, the train discharges gradually diminished and became minimal over the placental bed. This mixture of restricted asynchronous electrical activity, which is characteristic of the progesterone block (Csapo \& Takeda, 1965), allowed only small and irregular intra-uterine pressure cycles, as illustrated in Fig. 2. The effect of oxytocin, also shown in Fig. 2, was to improve the electrical activity, both in the horn which had already delivered and at the interplacental sites of the intact horn, while having little effect upon the activity recorded from the placental sites. Oxytocin in the experimental horn generated increased intra-uterine pressure at high frequency whereas its inconsistent effect on different areas of the intact horn did not improve the pressure in that horn.

These results demonstrate a functional asymmetry between the intact pregnant and placenta-dislocated uterine horns of the bicornuate rat uterus as well as between the anti-placental and placental regions of the intact pregnant horn itself. The functional asymmetry of the intact horn apparently depends on the topographical relationship of the uterine area to functional placentae. The question is whether this asymmetry is directly related to progesterone, or whether the placental effect is mediated by other factors, for example, structural differences between uterine regions. Our contention that this placentadependent functional asymmetry is causally related to the direct effect of progesterone, is based on the following observations.

It has been shown (Takeda, personal communication) that when the rat uterus is excised at the end of pregnancy and strips are suspended in an isolated organ bath, the electrophysiological difference between placental and non-placental sites gradually disappears. This in vitro evolution of activity in the placental regions cannot be accounted for by structural changes but rather by the metabolism of progesterone in the endocrinologically unsupported uterus (Wiest, 1963). Such a rapid in vitro deterioration of the myometrial block has also been demonstrated in the rat uterus on the 10th day of pregnancy by a quick evolution of the train discharge and a shift in the 'strength-duration' curve (threshold) as a function of the duration of in vitro exposure (see Fig. 2, Csapo, 1968). 
Experiments are in progress in collaboration with Dr W. Wiest to measure the progesterone content of uterus, placenta and peripheral plasma in rats. The estimations are being carried out with the sensitive and specific 'double isotope derivative dilution' method of Wiest (1967). At present the number of results is insufficient for complete statistical analysis but various pointers are becoming apparent. In one series, rats were ovariectomized on the 17th day of pregnancy and the placentae in one horn were dislocated. Abortion from the placentadislocated horn began about $10 \mathrm{hr}$ later and the animals were killed $18 \mathrm{hr}$ after the operation for progesterone analysis. Results indicated not only a general progesterone withdrawal, but a difference between the intact and experimental horn. Thus the progesterone concentration was apparently greater in the intact horn than in the active horn which had aborted its foetuses. Progesterone therapy started at the time of ovariectomy prevented delivery from the placenta-dislocated horn and diminished the difference between the two horns in the concentration of progesterone. Additional results are needed, however, to establish the statistical significance of these findings.

It might be argued that the findings referred to earlier of synchronous electrical activity and high intra-uterine pressure cycles in the horn with dislocated placentae, were due to structural changes, i.e. to an increase in wall thickness and in the number of cells near the electrode, resulting from the shortening of this horn during the process of abortion. This seems unlikely, however, since it was shown (Csapo, Takeda \& Wood, 1963) that it is lengthening of myometrial fibres (up to an optimal value) induced by volume increase in the parturient rabbit uterus which increases the synchrony of train discharges and intra-uterine pressure.

Thus, in general, structural differences fail to account for the observed functional asymmetry of the uterus, whereas differences in progesterone concentration apparently do.

It is important to note here that there were significant exceptions to a consistent relationship between plasma and uterine progesterone concentrations. For example, in control rats the concentrations in the two tissues were similar and both decreased at the end of pregnancy. However, when rats were ovariectomized on day 16 and pregnancy was maintained until day 21 as a result of placental hypertrophy, the 'pre-partum' concentration of progesterone in the uterus was similar to that of controls, whereas the concentration in the plasma was about one-third of that in controls, the difference being significant with $P<0.001$. Hence it is not always possible to predict uterine from plasma concentrations and it would seem wiser always to make direct measurements in the relevant tissues.

\section{Experiments in pregnant rabbits}

We also examined the 'dispensibility' of the corpus luteum in pregnant rabbits and the potential of this animal to perform the 'luteo-placental shift' successfully. A total of fifty-two New Zealand White rabbits, with precisely dated pregnancies, were studied under experimental conditions previously described (Csapo \& Lloyd-Jacob, 1962). In the control animals (thirty-two in number), delivery was induced with a single i.v. dose of $100 \mathrm{mU}$ oxytocin, starting on day 30 of gestation. In case of induction failure, this treatment was repeated at intervals of $12 \mathrm{hr}$ until the animal delivered. Induced, rather than spontaneous, delivery was preferred in order to recover the majority of foetuses and placentae for immediate measurements.

The twenty experimental animals (with litters of less than eight; Fig. 3, numbers in double circles) were ovariectomized on the 18th or 19th day of $\vec{\varphi}$ gestation, i.e. 11-12 days after implantation. This if procedure terminates pregnancy within $48 \mathrm{hr}$ unless protection is guaranteed by progesterone therapy. In order to test for the placental compensatory potential of these ovariectomised rabbits, a 'transient decremental' progesterone therapy was given (Fig. 3) in order to sustain pregnancy during the initial period of predicted placental compensatorg effort. Treatment began at the time of ovariectom at the $4 \mathrm{mg} /$ day level. The hormone was given in oibo intramuscularly, in two divided daily doses. It was decreased by $1 \mathrm{mg}$ every $2 \mathrm{nd}$ day and tapered off to the level of $0.5 \mathrm{mg}$ /day on days $26-27$.

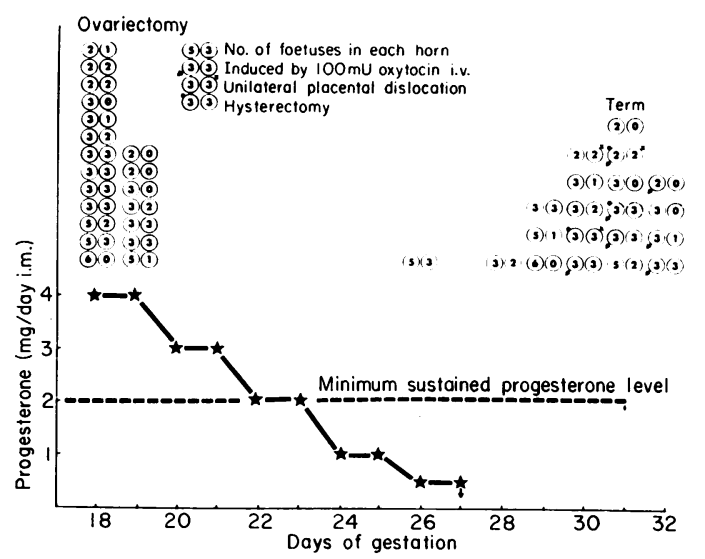

FIG. 3. Showing the times of ovariectomy, decremental progesterone therapy and subsequent delivery of foetuses in twenty rabbits. Litter size is shown by the numbers in circles. Delivery was spontaneous unless otherwise indicated. The broken horizontal line shows the minimum level of continued progesterone administration necessary for the maintenance of pregnancy. 
TABLE 1. The effect in pregnant rabbits of ovariectomy + transient, decremental progesterone treatment on foetal and placental weights at delivery, as compared with normal controls

\begin{tabular}{|c|c|c|c|c|}
\hline & \multicolumn{2}{|c|}{ Control } & \multicolumn{2}{|c|}{ Experimental } \\
\hline & Foetus (g) & Placenta (g) & Foetus (g) & Placenta (g) \\
\hline $\begin{array}{l}\text { Day } 30 \\
\text { Day } 31 \\
\text { Day } 32\end{array}$ & $\begin{array}{l}40 \pm 1 \cdot 0 \\
47 \pm 0 \cdot 8 \\
50 \pm 1 \cdot 5\end{array}$ & $\begin{array}{l}4 \cdot 5 \pm 0 \cdot 2(9) \\
5 \cdot 1 \pm 0 \cdot 1(17) \\
5 \cdot 1 \pm 0 \cdot 7(6)\end{array}$ & $\begin{array}{l}53 \pm 1 \cdot 9 \\
60 \pm 1 \cdot 2 \\
75 \pm 2 \cdot 2\end{array}$ & $\begin{array}{c}10 \cdot 1 \pm 0 \cdot 5(5) \\
7 \cdot 7 \pm 0 \cdot 5(6) \\
9 \cdot 0 \pm 0 \cdot 4(4)\end{array}$ \\
\hline Total average & $46 \pm 0.6$ & $5 \cdot 0 \pm 0 \cdot 1$ & $68 \pm 1 \cdot 2$ & $9 \cdot 1 \pm 0.3(15)$ \\
\hline
\end{tabular}

The figures in parentheses represent the number of pregnant rabbits studied.

The difference in both foetal and placental weights between control and experimental groups, at 30,31 and 32 days, as well as the total average difference is highly significant $(P<0.001)$.

The four experimental animals, delivered prematurely between days 28 and 30 , had an average placental value of $5.4 \pm 0.3 \mathrm{~g}$. This value is similar to that of the controls, but significantly different $(P<0.001)$ from that of the fifteen experimental animals.

In fifteen out of twenty ovariectomized rabbits, pregnancy was maintained until days $30-32$, that is, several days after the cessation of progesterone treatment. When delivery occurred in these fifteen, either spontaneously or induced by oxytocin (Fig. 3, see arrows), oversized foetuses and placentae were discharged (Table 1). In three animals pregnancy had to be terminated by hysterectomy (Fig. 3, black dots on circles) because oxytocin failed to induce complete delivery. Thus the combined effect of ovariectomy + transient decremental progesterone therapy (so designed as to imitate the gradual luteal progesterone withdrawal of human pregnancy) created, in the majority of animals, a regulatory condition in which the ovaries became dispensible for the maintenance of pregnancy.

Table 1 shows the average foetal and placental weights (in $\mathbf{g} \pm \mathrm{SD}$ ) of the thirty-two control and nineteen experimental rabbits as a function of the day of delivery. On days 30,31 and 32 the average foetal and placental weights of the control and experimental animals are significantly different with $P<0.001$ and the averages for the 3 days combined are also significantly different with $P<0.001$. The average placental weight of those four experimental rabbits which delivered on days 28 and 29 is similar to that of the controls but significantly lower $(P<0.001)$ than that of the remaining fifteen experimental rabbits which maintained pregnancy for a longer period. The rabbit which delivered on day 26 likewise had placentae of comparable weight to controls.

It is evident, therefore, that the maintenance of pregnancy in ovariectomized rabbits (transiently supported during initial placental compensatory effort by decremental progesterone therapy) coincided with marked placental hypertrophy and accelerated pre-natal development of the foetus.
This and the additional observation that those five rabbits which failed in placental hypertrophy delivered prematurely, suggest that placental hypertrophy and the maintenance of pregnancy are causally related. Thus effective luteo-placental shift in rabbits and rats, aiming at the maintenance of pregnancy in the absence of luteal function, apparently depends on the success of placental compensatory effort.

In three of the rabbits in the experimental group, in addition to the ovariectomy and decremental progesterone therapy, the placentae were dislocated in one horn on day 24. While all three animals carried pregnancy until days $30-31$ in the intact control horn (Fig. 3), they all aborted, completely or partially, within $48 \mathrm{hr}$ from the horn in which the placentae had been dislocated. This simultaneous occurrence in the rabbit (as in the rat) of unilateral abortion from one uterine horn and intact pregnancy in the other, is evidence of a local, unilateral, placenta-dependent controlling factor. Whether this factor in the rabbit is progesterone (as it appears to be in the rat) is now under investigation.

\section{Conclusions}

The experiments presented show that in pregnant rabbits and rats, as in human patients, the corpora lutea are dispensible provided that the animal can effectively accomplish compensatory placental hypertrophy. This change, the luteo-placental shift, results in such modifications in myometrial regulation as to allow arcuate or bicornuate uterine horns to function independently from one another and deliver their contents at different times. Thus Nature's puzzle, the delivery of human twins several weeks apart, which led to the concept of the 'local' effect of placental progesterone (Csapo, 1956, 1961), has been reproduced experimentally. This concept has gained considerable support from the findings 
that both the electrophysiological and mecanicalh functional asymmetry of the uterus may be induced by unilateral placental dislocation, that this procedure results in a reduction in uterine progesterone concentration and that this fall in uterine progesterone can be balanced by progesterone substitution therapy preventing the discharge of the dislocated conceptuses. The premises stated in the Introduction are supported, therefore, by experimental evidence.

The significance of placental hypertrophy in an effective luteo-placental shift is also demonstrated. That the placental dominated human pregnancy, as opposed to the luteal dominated rat and rabbit pregnancy, involves marked placental hypertrophy is indicated by the low foetal/placental ratio: $7 \cdot 3$ in contrast to 9.8 and 11.7 , respectively. Premature delivery in ovariectomized rats focused upon the significance of this ratio by revealing that when placental hypertrophy fails, placental retention and the lowering of the foetal/placental ratio becomes the ultimum refugiens in the maintenance of pregnancy. When all the experiments described are considered, the control over placental growth emerges as the outstanding problem of obstetrics and its supporting disciplines. This regulatory mechanism must be understood before any claims are justified for a predictable control over the function of the pregnant uterus and the protection of pre-natal life.

\section{Acknowledgments}

The author is grateful to Dr Martti Pulkkinen and Elise Csapo for assistance during the experiments, for the processing of the data and for statistical analysis and to Dr Brenda M. Schofield and Dr David Porter for the benefit of discussions.

This work was supported by Grants HDO1478 and 5-K6-HD 20,169 from the National Institute of Health, United States Public Health Service and the Sunnen Foundation.

\section{References}

Bengtsson, L.PH. \& CsaPo, A.I. (1962) Oxytocin response, withdrawal and reinforcement of defense mechanism of the human uterus at midpregnancy. Amer. J. Obstet. Gynec. 83, 1083.

CsaPo, A.I. (1956) Progesterone 'block'. Amer. J. Anat. 98, 273.

Csapo, A. (1961) Defence mechanism of pregnancy. Progesterone and the Defence Mechanism of Pregnancy. Ciba Foundation Study Group No. 9.
Csapo, A.I. (1967) The 'ovariectomy syndrome' and the initiation of labor. Proceedings of the International Symposium on Prenatal Life, Detroit (E.d. by H.C. Hack). Thomas, Springfield, Illinois.

CsAPO, A.I. (1968) The electrophysiological manifestations of the progesterone effect on the uterus. The International Encyclopedia of Pharmacology and Therapeutics (Ed. by H. Tausk) (In press).

CsaPo, A.I. \& Lloyd-Jacob, M.A. (1962) Placenta, uterine volume, and the control of the pregnant uterus in rabbits. Amer. J. Obstet. Gynec. 83, 1073.

Csapo, A., Pinto-Dantas, C.A., Kerenyi, T., De Sousa, O., Darze, E., Lindblade, J. \& KaO, S.M. (1966) Pro- ळे gesterone and Myometrial Activity. Excerpta Medica International Congress Series No. 133. Proc. 5th World Congress on Fertility and Sterility, Stockholm, p. 429.

Csapo, A. \& Sauvage, J. (1968) The evolution of uterine activity during human pregnancy. Acta scand. Obstet. Gynec. (In press).

CSAPO, A.I. \& TAKEDA, H. (1965) Effect of progesterone on the electric activity and intrauterine pressure of pregnant and parturient rabbits. Amer. J. Obstet. Gynec. 91, 221.

Csapo, A.I., TAKeda, H. \& Wood, C. (1963) Volume and or activity of the parturient rabbit uterus. Amer. J. Obstet. Gynec. 85, 813.

Csapo, A. \& Wood, C. (1968) The endocrine control of the initiation of labor in the human. Rec. Advanc. Endocrinol. (Ed. by V.H.T. James) (In press).

Hoffman, B.F., Cranefield, P.F., Lepeschkin, E., SuraWICZ, B. \& HerRLICH, H.C. (1959) Comparison of cardiac monophasic action potentials recorded by intracellular and suction electrodes. Amer. J. Physiol. 196, 1297.

Johansson, E.D.B., NeILl, J.D. \& KNOBIL, E. (1968) Periovulatory progesterone concentration in the peripher plasma of the rhesus monkey with a methodologic not on the detection of ovulation. Endocrinology, 82, 143.

Porter, D.G., Becker, R. \& CSAPo, A.I. (1968) On the mechanism of action of intra-amniotic hypertonic saline treatment in rabbits. J. Reprod. Fert. 17, 433.

Runnebaum, B. \& Zander, J. (1967) Progesterone in human peripheral blood in pre-ovulatory period of menstrual cycle. Acta endocr. (Kbh.), 55, 91.

VAN DER MOLEN, H.J. \& GROEN, D. (1965) Determination of progesterone in human peripheral blood using gasliquid chromatography with electron capture detection. J. clin. Endocr. 25, 1625.

VAN DER VIES, J. \& FeENSTRA, H. (1967) The effects of ovarian hormones on the placenta of rats. Acta endocr. (Kbh.), Suppl. 119, p. 235.

WIEST, W.G. (1963) In vitro metabolism of progesterone and $20 \alpha$-hydroxypregn-4-en-3-one by tissues of the female rat. Endocrinology, 73, 310.

WIEst, W.G. (1967) A double isotope derivative assay for progesterone and $20 \alpha$-hydroxypregn-4-en-one. Steroids $10,257$.

WiLSON, K.M. (1937) Pregnancy complicated by ovarian and parovarian tumors. Amer. J. Obstet. Gynec. 34, 977. 